\title{
Reduced Periprocedural Analgesia After Replacement of Water for Injection with Glucose 5\% Solution as the Infusion Medium for ${ }^{90}$ Y-Resin Microspheres
}

\author{
Karolin Johanna Paprottka ${ }^{1}$, Sebastian Lehner ${ }^{2}$, Wolfgang P. Fendler ${ }^{2}$, Harun Ilhan ${ }^{2}$, Axel Rominger ${ }^{2}$, Wieland Sommer ${ }^{1}$, \\ Dirk A. Clevert ${ }^{1}$, Mark op den Winkel ${ }^{3}$, Volker Heinemann ${ }^{4}$, and Philipp Marius Paprottka ${ }^{1}$ \\ ${ }^{1}$ Department of Clinical Radiology, LMU University of Munich, Munich, Germany; ${ }^{2}$ Department of Nuclear Medicine, LMU \\ University of Munich, Munich, Germany; ${ }^{3}$ Department of Hepatology, LMU University of Munich, Munich, Germany; \\ and ${ }^{4}$ Department of Oncology, LMU University of Munich, Munich, Germany
}

\begin{abstract}
The primary aim of our study was to compare the need for periinterventional on-demand analgesia when water for injection (WFI) was replaced with glucose $5 \%$ (G5) for ${ }^{90}$ Y-resin microsphere administration. Methods: Forty-one patients who received 77 radioembolization procedures with G5 (2014-2015) were retrospectively matched with 41 patients (77 radioembolization procedures) who received radioembolization with WFI (2011-2014) at our center. The need for on-demand pain medication was chosen as an objective and accessible measure of periprocedural pain experienced by patients. Results: Patients were well matched according to sex, age, tumor type and involvement, and prior antiangiogenic therapies. Periinterventional analgesic requirements were significantly lower for radioembolization procedures performed with G5 than WFI: 5 of 77 (6.5\%) versus 29 of 77 (37.7\%), $P \leq 0,001$, respectively. Early stasis (defined as slowed antegrade flow, before total vascular stasis) occurred in 12 of $154(7.8 \%)$ radioembolization procedures overall and was not different $(P \leq 0.229)$ between the 2 groups $(4 / 77$ [5.2\%] vs. 8/77 [10.4\%]). Conclusion: Slow pulsatile administration of ${ }^{90}$ Y-resin microspheres with WFI is associated with a low rate of stasis. Replacement of WFI with G5 significantly reduces the need for periprocedural analgesia. These data favor the use G5 for $90 Y$-resin microsphere implantation in daily practice.
\end{abstract}

Key Words: radioembolization (RE); water for injection (WFI); glucose 5\% (G5); peri-procedural analgesia; stasis rate

J Nucl Med 2016; 57:1679-1684

DOI: 10.2967/jnumed.115.170779

\footnotetext{
A

s the documented incidence of primary and metastatic liver cancers increases $(1,2)$, treatments using minimally invasive techniques are becoming more common. Radioembolization with ${ }^{90} \mathrm{Y}$-resin microspheres is a blood flow-directed therapy enabling intraarterially infused microscopic microspheres (median diameter, $32.5 \mu \mathrm{m}$ ) to lodge within the terminal arteries close to the liver tumors, without passing through the capillary bed (diameter, $\leq 7-10 \mu \mathrm{m}$ ) and hence

Received Dec. 3, 2015; revision accepted May 6, 2016.

For correspondence contact: Karolin Johanna Paprottka, Department of Clinical Radiology, LMU University of Munich, Nussbaumstrasse 20, 80336 Munich, Germany.

E-mail: karolin.paprottka@med.Imu.de

Published online Jun. 3, 2016.

COPYRIGHT (C 2016 by the Society of Nuclear Medicine and Molecular Imaging, Inc.
}

avoiding systemic effects. As observed in explanted whole livers, clusters of ${ }^{90}$ Y-resin microspheres gradually assemble after administration and reduce the terminal blood flow but without stopping it completely (3).

The dual blood supply of the liver enables the selective treatment of hepatic malignancies via the blood supply-mainly from the hepatic artery-whereas the normal liver parenchyma is perfused mostly by the portal circulation (4). Consequently, a high radiation dose can be delivered safely and effectively to primary and secondary liver tumors by radioembolization, with relative sparing to normal liver tissue $(5-10)$. Radioembolization has been evaluated as both a first-line therapy $(11,12)$ and in the salvage setting for chemoresistant/refractory metastatic colorectal cancer (13-16) and is a recommended salvage treatment by the clinical practice guidelines of the European Society of Medical Oncology (17). Radioembolization has also been extensively evaluated in hepatocellular carcinoma $(18,19)$, and new data are emerging for the effectiveness of this treatment in intrahepatic cholangiocarcinoma (9), as well as liver-predominant metastatic neuroendocrine tumors (20), breast cancer (21), and ocular melanoma (22). Uncommon side effects of radioembolization include ulceration or perforation of the stomach or duodenum, cholecystitis, and pancreatitis, due to the nontargeted deposition of microspheres $(23,24)$. After a learning period, the incidence of nontargeted deposition is falling under $<1 \%$ to $<3.5 \%$, as published in the literature (13). Periinterventional complete stasis and potential consequent reflux is thought to increase the risk of nontargeted microsphere deposition. Hence, it is important to administer microspheres using the correct injection technique (slow and pulsatile) and to monitor the radioembolization procedure using real-time fluoroscopy and injectable nonionic contrast medium to ensure the correct catheter position and assessment of vessel behavior (vasoconstriction/spasm) and possible early stasis.

Because ${ }^{90} \mathrm{Y}$ is bound to the resin polystyrene microspheres through an ion exchange mechanism, sterile water for injection (WFI/because of its nonionic properties) has been used traditionally as the vehicle for radioembolization with ${ }^{90}$ Y-resin microspheres. Although the published safety and efficacy profile of radioembolization (using WFI as the vehicle for the delivery of the microspheres) is consistently favorable, it has recently been proposed that WFI might be replaced by a more optimal vehicle.

Studies, both in animals (25) and in humans (26), have shown that WFI, injected into a temporarily isolated segment of a blood vessel, removes the arterial endothelium. Vessel constriction/spasm 
was observed after 15-min perfusion of distilled water at $5 \mathrm{~mL} / \mathrm{min}$. For this reason, a slow, pulsatile injection of microspheres in WFI may prevent osmotic injuries to the endothelium, because it allows the dilution of the hypo-osmolar WFI in the blood, ultimately leading to early stasis (in few patients). The endothelial injury and vasoconstriction/vasospasm might cause discomfort/ pain (in many patients) during and after radioembolization, although the degree of endothelial injury and vasospasm may not correlate with patients' perception of pain. This pain is managed with analgesics.

Published reports indicate that, for the few patients experiencing early stasis with ${ }^{90}$ Y-resin microspheres, 3-mo response rates appear not to be altered (as assessed by imaging) (27). Although early stasis or flow reduction may occur in $20 \%-35 \%$ of procedures $(7,28)$, its link with the periprocedural pain is not well described in the literature.

Glucose 5\% (G5), also known as dextrose 5\% water, is a physiologically isotonic nonionic solution, which is now being evaluated as a vehicle for the infusion of the ${ }^{90}$ Y-resin microspheres instead of WFI. Our hypothesis is that the isotonic nature of G5 may prevent arterial endothelial injury and consecutive vasoconstriction and so may reduce the periprocedural need for painkillers.

The primary aim of our study was to compare the need for periinterventional on-demand analgesia when WFI was replaced with G5 for the administration of ${ }^{90}$ Y-resin microspheres (SIRSpheres; Sirtex Medical Ltd.).

The incidence of early stasis (defined as slowed antegrade flow, before total vascular stasis) and the calculated and delivered activity of ${ }^{90} \mathrm{Y}$ were also analyzed. Results were compared with a matched-pair analysis of prior radioembolization procedures using WFI as the vehicle; otherwise, the protocol for treatment was the same.

We chose on-demand analgesia because it is an objective and accessible measure of pain experienced by patients. By contrast, the evaluation of early stasis (defined as mildly slowed antegrade flow to just before total vascular stasis) is highly inter- and intraoperatordependent, with rates ranging between $20 \%$ and $35 \%$ in the literature $(7,27)$.

\section{MATERIALS AND METHODS} Board.

This retrospective analysis was approved by the Institutional Review

\section{Patient Selection}

The decision to treat patients with radioembolization was based on the published criteria (29) and the consensus of the multidisciplinary tumor board; all patients provided written informed consent. Between August 2014 and January 2015, 77 radioembolization procedures were performed in 41 patients using G5 as the vehicle for the delivery of ${ }^{90}$ Y-resin microspheres. Patients in the G5 group were matched with 41 patients from our database who had received 77 radioembolization procedures using WFI. Patients were matched according to sex, age, tumor type and involvement, and prior antiangiogenic therapies.

\section{Dosimetry}

Dose calculations were based on partition model, using exact liver and tumor volumes calculated by dedicated software application (OncoTREAT; MeVis).

\section{Radioembolization Procedure}

Arteriography was performed via a transfemoral approach. The presence of angiographically occult afferent extrahepatic arteries and the magnitude of hepatopulmonary shunting were evaluated by hepatic arterial injection of approximately $100 \mathrm{MBq}$ of ${ }^{99 \mathrm{~m} T \mathrm{~T}-\text { macroaggregated }}$ albumin ( $\left.{ }^{99 \mathrm{~m}} \mathrm{Tc}-\mathrm{MAA}\right)$ in the left, right, and sometimes segment 4 hepatic artery separately according to the planned catheter positions for radioembolization. Instead of systematic coil embolization during the ${ }^{99 m}$ Tc-MAA angiography, we favored the distal placement of the catheter at least $2 \mathrm{~cm}$ from the gastroduodenal artery (or other artery supplying blood to the gut) to prevent the nontarget distribution of microspheres. However, for centers starting radioembolization procedures, we strongly recommend coiling all the classic (such as gastroduodenal artery) and suspect collateral vessels before performing radioembolization.

The primary purpose of pretreatment ${ }^{99 \mathrm{~m}} \mathrm{Tc}-\mathrm{MAA}$ SPECT simulation was to evaluate extrahepatic deposition particles (in the stomach, pancreas, or duodenum), and if aberrant vessels were not identified by angiogram, we performed a pretreatment Dyna CT. If the catheter could not be placed within a sufficient safety margin $(\geq 2 \mathrm{~cm})$ to classic or suspected collaterals, vascular occlusion using microcoil embolization was always performed even if nontargeted deposition was not observed on ${ }^{99 m}$ Tc-MAA SPECT/CT. Other factors that determined the need for microcoil embolization were the quality of the antegrade flow in tumor afferent selected for radioembolization and presence of potential safety vessels (allowing coiling of the considered vessel). ${ }^{90}$ Y-resin microspheres, suspended in G5 or WFI, were injected under intermittent fluoroscopic visualization, alternating with contrast medium, to assess antegrade hepatic arterial flow.

${ }^{90}$ Y-resin microspheres were administered as a lobar or sequential lobar treatment (with an interval of 4-6 wk between procedures) depending on the distribution of tumors within the liver. In all cases, the left, right, and (if applicable) segment 4 hepatic artery were catheterized and treated separately, to improve safety. Radioembolization was never performed from the common or proper hepatic artery. SPECT (or SPECT/CT) scans were obtained within $24 \mathrm{~h}$ to confirm targeted deposition of ${ }^{90}$ Y-resin microspheres (Bremsstrahlung SPECT).

\section{Periprocedural Medication}

Details of the periprocedural medication are presented in Table 1.

\section{Statistics}

A commercial statistical software package (SPSS 22.0 for Apple; SPSS) was used. Statistical analysis was performed using the $\chi^{2}$ test with a significance level fixed at 0.05 .

\section{RESULTS}

\section{Patients' Characteristics}

Both groups were well balanced based on the 5 criteria for the matched-paired analysis as well as tumor involvement (defined as a percentage of whole liver) and liver-to-lung shunting (Table 2).

\section{Periprocedural Analgesia}

Periinterventional analgesic requirements were significantly lower for radioembolization procedures performed using G5 than for radioembolization procedures using WFI: 5 of 77 (6.5\%) versus 29 of 77 (37.7\%), $P \leq 0.001$, respectively. This corresponded to an $83 \%$ reduction in the use of on-demand pain medication with G5 (Fig. 1).

\section{Early Stasis}

In total, early stasis was recorded in 12 of $154(7.8 \%)$ radioembolization procedures: 4 of 77 (5.2\%) procedures using G5 
TABLE 1

Periprocedural Medication

\begin{tabular}{|c|c|c|c|c|}
\hline Scheme & Medication & Schedule & Start & Treatment duration \\
\hline \multirow[t]{2}{*}{ REILD prophylaxis } & $\begin{array}{l}\text { Ursodesoxycholic acid, } \\
250 \text { mg }\end{array}$ & $1-0-1$ & $\begin{array}{l}\text { On day of } \\
\text { radioembolization }\end{array}$ & $12 \mathrm{wk}$ \\
\hline & Methylprednisolone, $8 \mathrm{mg}$ & $1-0-0$ & At discharge & $12 \mathrm{wk}$ \\
\hline \multirow[t]{7}{*}{$\begin{array}{l}\text { Periradioembolization } \\
\text { schedule }\end{array}$} & Ibuprofen, 600 mg & On-demand & $\begin{array}{l}\text { During radioembolization/after } \\
\text { radioembolization }\end{array}$ & Angiosuite/in-patient stay \\
\hline & Piritramid, $7.5 \mathrm{mg}$ & On-demand & $\begin{array}{l}\text { During radioembolization/after } \\
\text { radioembolization }\end{array}$ & Angiosuite/in-patient stay \\
\hline & Ciprofloxacin & Case-by-case & During radioembolization & Angiosuite \\
\hline & Pantoprazole & Case-by-case & During radioembolization & Angiosuite \\
\hline & Corticosteroids & Case-by-case & During radioembolization & Angiosuite \\
\hline & $\begin{array}{l}\text { Dimenhydrinate, } 120 \mathrm{mg} \\
\text { nicotinic acid, } 75 \mathrm{mg} \\
\text { pyridoxine, } 30 \mathrm{mg}\end{array}$ & Case-by-case & After radioembolization & In-patient stay \\
\hline & Metamizole & Case-by-case & After radioembolization & In-patient stay \\
\hline
\end{tabular}

versus 8 of $77(10.4 \%)$ procedures using WFI ( $\chi$-quadrat test $P=$ 0.229) (Fig. 2; Table 3).

\section{Radiation Absorbed Dose Reduction in Patients \\ Experiencing Early Stasis}

For patients receiving radioembolization with $\mathrm{G} 5$, the mean radiation absorbed dose reduction due to early stasis was $20.2 \%$ ( $n=3$ patients); a fourth patient with early stasis had no radiation absorbed dose reduction. For patients receiving radioembolization with WFI, early stasis led to a reduction of the applied radiation absorbed dose in 6 of 8 patients with stasis: mean total radiation-absorbed dose reduction, $25.8 \%(n=6)$. Because of a lower rate of stasis, the relative applied-to-calculated radiation absorbed dose was greater in the G5 group (98.3\%) than the WFI group (93.4\%) (Table 2).

TABLE 2

Baseline Patient Characteristics and Calculated and Applied ${ }^{90}$ Y Activity for Radioembolization in G5 and WFI Groups

\begin{tabular}{|c|c|c|}
\hline Characteristic & G5 $(n=41)$ & WFI $(n=41)$ \\
\hline Median age $(y)$ & 66 & 68 \\
\hline Sex (male:female) & $25: 16$ & $25: 16$ \\
\hline \multicolumn{3}{|l|}{ Primary tumor etiology } \\
\hline Hepatocellular carcinoma & 19 & 19 \\
\hline Intrahepatic cholangiocarcinoma & 5 & 5 \\
\hline Colorectal cancer & 6 & 6 \\
\hline Neuroendocrine tumor & 6 & 6 \\
\hline Gastrointestinal stromal tumor & 1 & 1 \\
\hline Breast cancer & 1 & 1 \\
\hline Ocular melanoma & 1 & 1 \\
\hline Ovarian cancer & 1 & 1 \\
\hline Adenocarcinoma (of undefined origin) & 1 & 1 \\
\hline Mean lung shunt fraction $\pm \mathrm{SD}(\%)$ & $5.9 \pm 2.7$ & $5.2 \pm 2.3$ \\
\hline No. of prior antiangiogenic therapies & 4 & 4 \\
\hline \multicolumn{3}{|l|}{ Tumor involvement (\%) } \\
\hline Mean \pm SD & $33.5 \pm 22.5$ & $29.2 \pm 20.4$ \\
\hline Median & 30 & 28 \\
\hline \multicolumn{3}{|l|}{${ }^{90} \mathrm{Y}$ activity (MBq) } \\
\hline Mean & Calculated, 1,620/applied, 1,593 & Calculated, 1,558/applied, 1,455 \\
\hline Median & Calculated, 1,633/applied, 1,537 & Calculated, 1,600/applied, 1,502 \\
\hline SD & Calculated, 390/applied, 406 & Calculated, 369/applied, 380 \\
\hline
\end{tabular}




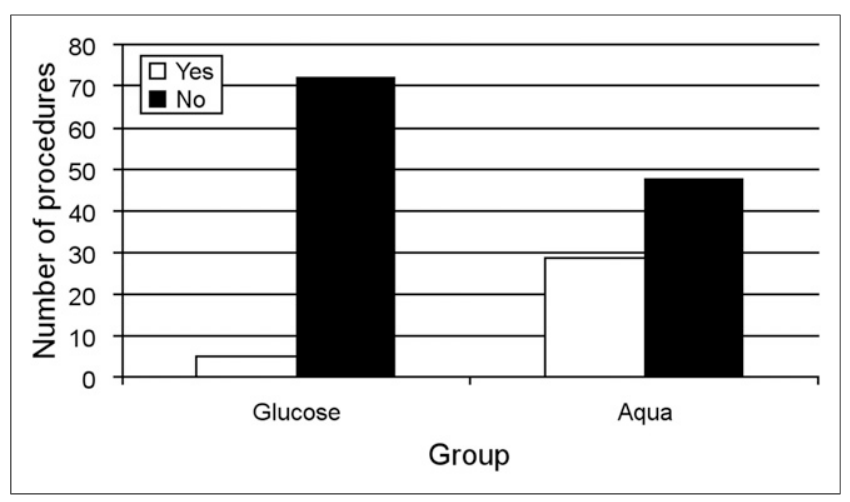

FIGURE 1. On-demand analgesic requirements after radioembolization using G5 as vehicle ( $n=77$ procedures) or WFI ( $n=77$ procedures).

\section{Antiangiogenics and Early Stasis}

The low number of patients receiving antiangiogenics $(n=4$ in each arm) before radioembolization prohibited any conclusions to be drawn (as only 1 of 8 patients had stasis).

\section{DISCUSSION}

Since its initial commercialization in 1998 in Australia, ${ }^{90} \mathrm{Y}-$ resin microspheres have been used in more than 50,000 radioembolization procedures, mainly using WFI as a vehicle for the implantation. To improve on the administration protocol, physicians - at first from the United States and now worldwideproposed replacing WFI with dextrose $5 \%$ (called glucose $5 \%$ in Europe), an isotonic nonionic solution, when microspheres are infused. Our data support the evidence that G5 can significantly reduce the requirement for on-demand analgesia during and after the radioembolization procedure from $37.7 \%$ to $6.5 \%$. This corresponds to an $83 \%$ decrease in pain medication. In other words, among the patients who might have requested painkillers with WFI, 4 patients of 5 will no longer require analgesia when G5 is used as the infusion solution.

Until now, there has been only 1 recently published paper dealing with this topic. Ahmadzadehfar et al. (30) analyzed 104 radioembolization procedures on 78 patients (41 patients with sterile water vs. 37 patients with G5) and observed that G5 procedures were associated with a significantly lower incidence of stasis ( $28 \%$ vs. $11 \%$ procedures; $P=0.02)$ and mild-tomoderate upper abdominal pain during the procedure (1.8\% vs. $44 \%$ procedures; $P<0.0001)$. The reduced stasis rate led to significantly more procedures for which the whole prescribed activity was administered (G5: $85 \%$ vs. $22 \% ; P<0.0001$ ). Additionally, a significantly higher proportion of the calculated activity was administered with G5: $96.1 \% \pm 11.0 \%$ versus $77.4 \% \pm 24.3 \%$ $(P<0.0001)$ (30). These results deviate from ours: although our stasis rate with sterile water $(10.4 \%)$ had already been lower than theirs using G5 (11\%), operator differences during the administration (most likely speed of injections) could be responsible for this finding. Although our results regarding the early stasis were not significant, we could observe a trend to lower rates using G5 (5.2\%) versus WFI (10.4\%).

The reduction of the mild-to-moderate upper abdominal pain during the procedure correlates with our results of the reduced requirement for on-demand analgesia during and after the radioembolization. Additionally, after informal discussion with interventional radiologists across Europe, the consensus was that periprocedural pain is generally mild, transient, and manageable, but nevertheless our experience of pain medication use in approximately $40 \%$ of radioembolization procedures appears to be compatible with the daily practice experience at other centers. Our study provides evidence for the belief, shared by the interventional radiology community, that the measure of ondemand analgesia during radioembolization is a clinically relevant endpoint.

The initial aim of the physicians who tested G5 was not to improve patients' comfort but to reduce the incidence of early stasis, so that calculated activity might be more efficiently administered. Concerns over stasis were strongly influenced by a study by Murthy et al. from the University of Texas, Houston, which reported that the delivered activity was less than the prescribed dose in 6 of the 17 procedures (35\%) (31). Unfortunately, this drew attention away from the other interesting finding in this paper, namely that the prescribed versus delivered median doses were as follows: right lobe, 1.584 versus $1.547 \mathrm{GBq}(42.8$ vs. $41.8 \mathrm{mCi}$ ) (difference, 2.3\%); left lobe, 2.131 versus $1.931 \mathrm{GBq}$ (26.3 vs. $20.6 \mathrm{mCi}$ ) (difference, $21.7 \%$ ); and whole liver, 0.973 versus $0.762 \mathrm{GBq}$ (57.6 vs. $52.18 \mathrm{mCi}$ ) (difference, 9.4\%). Second, the effect on efficacy of the treatment secondary to the inability to deliver the prescribed dose cannot be elucidated from such a small cohort of patients.

More recently (2014), Piana et al. (27) from Thomas Jefferson University published an article on 71 patients (128 radioembolization with ${ }^{90}$ Y-resin microspheres) treated between January 2007 and February 2010 (and therefore probably treated with WFI). This more comprehensive analysis showed that, despite an incidence of early stasis in approximately $20 \%$ of procedures, stasis did affect initial imaging outcomes (intrahepatic progressive disease vs. partial response and stable disease at $3 \mathrm{mo}$ ).

The reporting of early stasis (defined as slowed antegrade flow to just before total vascular stasis) appears to be highly inter- and intraoperator-dependent, with low rates reported in our study, which were not significantly influenced by the infusion medium.

We would recommend the slow, pulsatile infusion of microspheres to reduce the likelihood of stasis. The relatively low rates of stasis compared with the need for on-demand analgesia

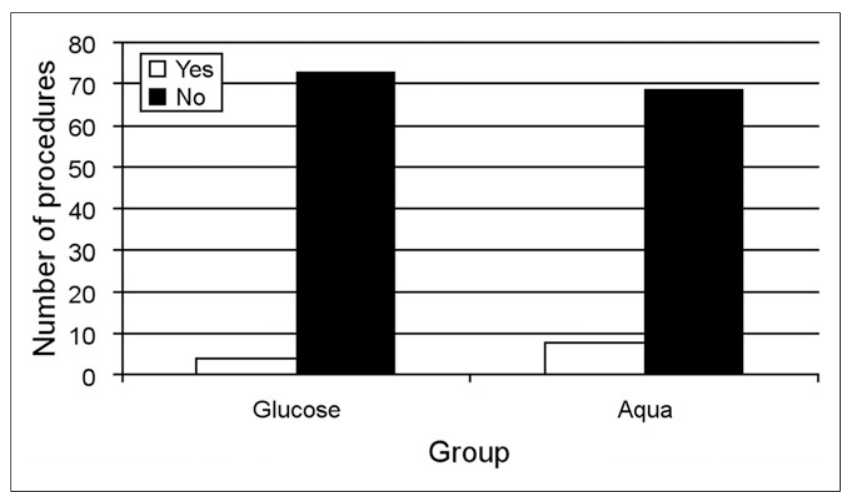

FIGURE 2. Occurrence of early stasis (slowed antegrade flow, before total vascular stasis has been reached) after radioembolization using G5 as a vehicle ( $n=77$ procedures) or WFI ( $n=77$ procedures). 
TABLE 3

Frequency of Early Stasis During Radioembolization, Expressed as Percentage of Number of Radioembolization Procedures with G5 $(n=77)$ or WFI $(n=77)$

\begin{tabular}{ccccc}
\hline Stasis & $\begin{array}{c}\text { Total } \\
(n=154)\end{array}$ & $\begin{array}{c}\text { G5 } \\
(n=77)\end{array}$ & $\begin{array}{c}\text { WFI } \\
(n=77)\end{array}$ & $P$ \\
\hline Early stasis & $12(7.8 \%)$ & $4(5.2 \%)$ & $8(10.4 \%)$ & 0.229 \\
$\begin{array}{c}\text { No early } \\
\text { stasis }\end{array}$ & 142 & $73(94.8 \%)$ & $69(89.6 \%)$ & \\
\hline
\end{tabular}

suggest that other mechanisms such as vasospasms (caused by endothelial injury) due to insult driven by the hypo-osmalarity of the infusion medium (with WFI) may be the primary mechanism of intraprocedural pain. This insult to the vasculature can be significantly reduced by the use of more isotonic infusion solutions.

Another concern of the physicians is that stasis during the infusion on the ${ }^{90}$ Y-resin microspheres may increase the potential risk of radiolabeled microspheres reflux, leading to unwanted deposition in nontargeted organs (stomach, pancreas, or duodenum). The accumulation of scientific data since 1998 has clearly demonstrated that ${ }^{90}$ Y-resin microspheres are a highly developed medical device requesting the centers to go through a learning curve to master the implantation process. Once the center has passed this learning period, the incidence of nontargeted deposition does not exceed $1 \%-3.5 \%$ as published in the literature (13).

Our work has some important limitations. First, it is a nonrandomized, retrospective, monocentric investigation. Nevertheless, in the absence of data from a randomized-controlled trial, a matched-paired analysis represents the highest methodologic standard applicable to generate comparative data on this subject. Second, the learning curve of the interventional radiologists team during the time frame covered by this study could introduce a possible bias when comparing historical data with WFI compared with current treatment with G5. However, radioembolization is performed by a highly experienced core team at our center who have treated a large number of patients with radioembolization year-on-year since 2002 . Therefore, any bias regarding this issue might be ruled out. Third, the treatment cohort was also a heterogeneous population, which limits the ability to generalize the findings to specific tumor entities. However, our study represents a typical radioembolization cohort and so would to the most appropriate setting for assessing the utility of G5 for ${ }^{90}$ Y-resin microsphere implantation in daily practice. A further prospective study would be valuable to compare some technical parameters of the ${ }^{90} \mathrm{Y}$-resin microspheres' administration, such as the time to perform the infusion and the volume of G5 or WFI and contrast medium infused.

\section{CONCLUSION}

We showed that the slow and pulsatile administration of the ${ }^{90}$ Y-resin microspheres with WFI is associated with a low rate of stasis. The replacement of WFI with G5 significantly reduces the need for periprocedural analgesia. These data favor the use G5 for ${ }^{90}$ Y-resin microspheres implantation in daily practice.

\section{DISCLOSURE}

The costs of publication of this article were defrayed in part by the payment of page charges. Therefore, and solely to indicate this fact, this article is hereby marked "advertisement" in accordance with 18 USC section 1734 . No potential conflict of interest relevant to this article was reported.

\section{REFERENCES}

1. El-Serag HB. Hepatocellular carcinoma: recent trends in the United States. Gastroenterology. 2004;127:S27-S34.

2. Jemal A, Murray T, Ward E, et al. Cancer statistics, 2005. CA Cancer J Clin. 2005;55:10-30.

3. Kennedy AS, Nutting C, Coldwell D, Gaiser J, Drachenberg C. Pathologic response and microdosimetry of ${ }^{90} \mathrm{Y}$ microspheres in man: review of four explanted whole livers. Int J Radiat Oncol Biol Phys. 2004;60:15521563.

4. Cohen AD, Kemeny NE. An update on hepatic arterial infusion chemotherapy for colorectal cancer. Oncologist. 2003;8:553-566.

5. Jakobs TF, Hoffmann RT, Tatsch K, Trumm C, Reiser MF. Therapy response of liver tumors after selective internal radiation therapy [in German]. Radiologe. 2008;48:839-849.

6. Jakobs TF, Hoffmann RT, Tatsch K, Trumm C, Reiser MF, Helmberger TK. Developments and perspectives in radioablative techniques [in German]. Radiologe. 2007;47:1083-1088.

7. Kennedy AS, Coldwell D, Nutting C, et al. Resin ${ }^{90}$ Y-microsphere brachytherapy for unresectable colorectal liver metastases: modern USA experience. Int J Radiat Oncol Biol Phys. 2006;65:412-425.

8. Lin M, Shon IH, Wilson R, D'Amours SK, Schlaphoff G, Lin P. Treatment response in liver metastases following ${ }^{90} \mathrm{Y}$ SIR-spheres: an evaluation with PET. Hepatogastroenterology. 2007;54:910-912.

9. Hoffmann RT, Paprottka PM, Schon A, et al. Transarterial hepatic yttrium-90 radioembolization in patients with unresectable intrahepatic cholangiocarcinoma: factors associated with prolonged survival. Cardiovasc Intervent Radiol. 2012;35:105-116.

10. Sommer WH, Ceelen F, Garcia-Albeniz X, et al. Defining predictors for long progression-free survival after radioembolisation of hepatic metastases of neuroendocrine origin. Eur Radiol. 2013;23:3094-3103.

11. Gray B, Van Hazel G, Hope M, et al. Randomised trial of SIR-Spheres plus chemotherapy vs. chemotherapy alone for treating patients with liver metastases from primary large bowel cancer. Ann Oncol. 2001;12:17111720 .

12. Van Hazel G, Blackwell A, Anderson J, et al. Randomised phase 2 trial of SIRSpheres plus fluorouracil/leucovorin chemotherapy versus fluorouracil/leucovorin chemotherapy alone in advanced colorectal cancer. J Surg Oncol. 2004;88:7885 .

13. Bester L, Meteling B, Pocock N, et al. Radioembolization versus standard care of hepatic metastases: comparative retrospective cohort study of survival outcomes and adverse events in salvage patients. J Vasc Interv Radiol. 2012;23: 96-105.

14. Hendlisz A, Van den Eynde M, Peeters M, et al. Phase III trial comparing protracted intravenous fluorouracil infusion alone or with yttrium-90 resin microspheres radioembolization for liver-limited metastatic colorectal cancer refractory to standard chemotherapy. J Clin Oncol. 2010;28:3687-3694.

15. Cosimelli M, Golfieri R, Cagol PP, et al. Multi-centre phase II clinical trial of yttrium-90 resin microspheres alone in unresectable, chemotherapy refractory colorectal liver metastases. Br J Cancer. 2010;103:324-331.

16. Seidensticker R, Denecke T, Kraus P, et al. Matched-pair comparison of radioembolization plus best supportive care versus best supportive care alone for chemotherapy refractory liver-dominant colorectal metastases. Cardiovasc Intervent Radiol. 2012;35:1066-1073.

17. Van Cutsem E, Cervantes A, Nordlinger B, Arnold D. Metastatic colorectal cancer: ESMO Clinical practice guidelines for diagnosis, treatment and follow-up. Ann Oncol. 2014;25(suppl 3):iii1-iii9.

18. Sangro B, Carpanese L, Cianni R, et al. Survival after yttrium-90 resin microsphere radioembolization of hepatocellular carcinoma across Barcelona 
clinic liver cancer stages: a European evaluation. Hepatology. 2011;54:868878.

19. Salem R, Lewandowski RJ, Mulcahy MF, et al. Radioembolization for hepatocellular carcinoma using yttrium-90 microspheres: a comprehensive report of long-term outcomes. Gastroenterology. 2010;138:52-64.

20. Devcic Z, Rosenberg J, Braat AJ, et al. The efficacy of hepatic ${ }^{90} \mathrm{Y}$ resin radioembolization for metastatic neuroendocrine tumors: a meta-analysis. J Nucl Med. 2014;55:1404-1410.

21. Cianni R, Pelle G, Notarianni E, et al. Radioembolisation with ${ }^{90}$ Y-labelled resin microspheres in the treatment of liver metastasis from breast cancer. Eur Radiol. 2013;23:182-189.

22. Klingenstein A, Haug AR, Zech CJ, Schaller UC. Radioembolization as locoregional therapy of hepatic metastases in uveal melanoma patients. Cardiovasc Intervent Radiol. 2013;36:158-165.

23. Lau WY, Ho S, Leung TW, et al. Selective internal radiation therapy for nonresectable hepatocellular carcinoma with intraarterial infusion of $90 \mathrm{yttrium} \mathrm{mi-}$ crospheres. Int J Radiat Oncol Biol Phys. 1998;40:583-592.

24. Yip D, Allen R, Ashton C, Jain S. Radiation-induced ulceration of the stomach secondary to hepatic embolization with radioactive yttrium microspheres in the treatment of metastatic colon cancer. J Gastroenterol Hepatol. 2004;19: 347-349.
25. Tanimura A, Tanaka S, Kitazono M. Superficial intimal injury of the rabbit carotid artery induced by distilled water. Virchows Arch B Cell Pathol Incl Mol Pathol. 1986;51:197-205.

26. Sogo N, Wilkinson IB, MacCallum H, et al. A novel S-nitrosothiol (RIG200) causes prolonged relaxation in dorsal hand veins with damaged endothelium. Clin Pharmacol Ther. 2000;68:75-81.

27. Piana PM, Bar V, Doyle L, et al. Early arterial stasis during resin-based yttrium90 radioembolization: incidence and preliminary outcomes. HPB (Oxford). 2014; 16:336-341.

28. Moureau-Zabotto L, Turrini O, Resbeut M, et al. Impact of radiotherapy in the management of locally advanced extrahepatic cholangiocarcinoma. BMC Cancer. 2013;13:568.

29. Kennedy A, Nag S, Salem R, et al. Recommendations for radioembolization of hepatic malignancies using yttrium-90 microsphere brachytherapy: a consensus panel report from the radioembolization brachytherapy oncology consortium. Int J Radiat Oncol Biol Phys. 2007;68:13-23.

30. Ahmadzadehfar H, Meyer C, Pieper CC, et al. Evaluation of the delivered activity of yttrium-90 resin microspheres using sterile water and $5 \%$ glucose during administration. EJNMMI Res. 2015;5:54.

31. Murthy R, Habbu A, Salem R. Trans-arterial hepatic radioembolisation of yttrium-90 microspheres. Biomed Imaging Interv J. 2006;2:e43. 